\title{
Tn5-induced lipopolysaccharide mutations in Bordetella pertussis that affect outer membrane function
}

\author{
M. L. Turcotte, ${ }^{1}$ Denis Martin, ${ }^{2}$ Bernard R. Brodeur ${ }^{2}$ and Mark S. Peppler ${ }^{1}$
}

Author for correspondence: Mark S. Peppler. Tel: +1 403492 2304. Fax: +1 4034927521.

e-mail: mark.peppler@ualberta.ca

1 Department of Medical Microbiology and Immunology, University of Alberta, 1-69 Medical Sciences Bldg, Edmonton, Alberta, Canada T6G $2 \mathrm{H} 7$

2 Unite de recherche en vaccinologie, Centre de Recherche en Infectiologie, Centre Hospitalier Universitaire de Québec, Sainte-Foy, Québec, Canada G1V 4G2

\begin{abstract}
An LPSB-specific mAb was used to screen for ten Tn5 insertion mutants of Bordetella pertussis which have LPS which is phenotypically distinct from either wild-type LPSAB or LPSB. Silver-stained SDS-PAGE gels showed nine different LPS phenotypes, six of which contain two clinically undocumented LPS bands, designated IntA and IntB based on their proximity to the LPSA and LPSB bands, respectively. Binding assays with LPSA- and LPSB-specific mAbs established changes in epitope exposure for the various mutant LPS, both in cell-free form and as presented on the surface of whole cells. The possible involvement of a number of genes, both structural and regulatory, was indicated in production of the altered phenotypes. PFGE and Southern blotting showed that the Tn5 inserts of seven mutants mapped to a region of the $B$. pertussis chromosome shown previously to encode the bp/ gene products of LPS biosynthesis. Mutants MLT3, MLT5 and MLT8, however, mapped to distinctly different parts of the chromosome. In addition, mutants MLT2 and MLT3 contributed to an accelerated frequency in the appearance of avirulent phase organisms despite their Tn5 inserts being over $1000 \mathrm{bp}$ from the bvgIASR locus. The alterations in LPS structure in the mutants changed their reactivity to strain-specific mAbs and their sensitivity to hydrophobic and hydrophilic antibiotics.
\end{abstract}

Keywords: Bordetella pertussis, lipopolysaccharide mutants, outer membrane integrity

\section{INTRODUCTION}

Bordetella pertussis is the principal causative agent of whooping cough. The cell wall of B. pertussis, as in most Gram-negative organisms, is a complex matrix of interacting molecules. The bacterium is distinct from many other pathogens in its utilization of a number of unique toxins and virulence factors, many membraneassociated, in the overall pathogenicity of the disease (Weiss \& Hewlett, 1986). Characterization of the properties of any one isolated factor yields only limited data because of the collaborative nature of the many molecules involved in $B$. pertussis infection and immunity. Thus, the roles of many specific virulence factors have been examined through the creation of genetically

\footnotetext{
Abbreviations: DIG, digoxigenin; LPSA, slower-migrating LPS of $B$. pertussis which binds mAb BL-2; LPSB, faster-migrating LPS of $B$. pertussis which binds $m A b B L-8$; IntA, LPS migrating between LPSA and LPS $B$, but closer to LPSA; IntB, LPS migrating between LPSA and LPSB, but closer to LPSB.
}

mutated B. pertussis strains in which a certain toxin or factor has been deleted (Weiss et al., 1983; Khelef et al., 1994). The purpose of the present study was to use $\operatorname{Tn} 5$ insertion in a similar manner to study the effects of alterations of LPS on the integrity of the B. pertussis cell wall and thus the cell as a whole.

As a primary component of the outer membrane, the LPS of $B$. pertussis plays a major role in the many interactions which occur between host and pathogen during the processes of infection, disease and immunity. Neither the direct effects on cell function mediated by the receptor, barrier and molecular exclusion properties of LPS, nor the indirect consequences on pathogenicity of LPS interactions with other B. pertussis membraneassociated molecules have been determined because complete deletion of the LPS destroys the integrity of the cell wall.

Although the isolated LPS of $B$. pertussis exhibits many of the toxic and immunological properties of the LPS of enteric species (Chaby \& Caroff, 1988; Lasfargues et al., 
1993), the lack of commonly associated symptoms such as fever and septicaemia in clinical pertussis has resulted in some confusion as to the role of LPS in pathogenicity. Similarly, although implicated in numerous side-effects, the role of LPS has not been definitively established in the reactivity of the current whole-cell pertussis vaccine (Cody et al., 1981; Gold, 1988; Lasfargues et al., 1993). The properties of $B$. pertussis LPS in its natural environment, interacting with other components of the outer membrane, have yet to be fully explored.

Our intent was to investigate the effect of structural alterations of the LPS on the integrity and function of the $B$. pertussis outer membrane and thus, bacterial pathogenicity. In addition, we hoped to obtain data on the genetic regulation of the B. pertussis LPS biosynthetic pathway. The wild-type LPS of B. pertussis, while devoid of the extended O-side chains common in enteric bacteria, does exhibit a limited degree of heterogeneity (Chaby \& Caroff, 1988; LeDur et al., 1980; Peppler, 1984). Two bands are present on SDS-PAGE, a higher molecular mass component designated LPSA and a minor, lower molecular mass band designated LPSB (Caroff et al., 1990; Peppler, 1984). Caroff et al. (1990) established the source of this heterogeneity as the lack of three terminal amino sugars on the LPSB core (Caroff $e t$ al., 1990; Le Blay et al., 1996). These experiments were made possible by the existence of a less frequently isolated clinical strain of $B$. pertussis whose only LPS component is LPSB. We utilized a mAb recently developed against LPSB to screen $\mathrm{Tn} 5$ mutants of the wildtype LPSAB strain for LPS alterations (Archambault et al., 1991; Martin et al., 1992)

Our laboratory has produced by $\operatorname{Tn} 5$ insertion ten LPS mutants, which contain varying quantities of LPSA and LPSB as well as two clinically undocumented intermediate LPS types which we have designated IntA and IntB (respective to the band to which they are closest in molecular mass). This study deals with the phenotypic and genetic characterization of these mutants as well as the initial analysis of the effect of these mutations on outer membrane function. A genomic region of approximately $100 \mathrm{~kb}$ is responsible for the LPS phenotypes completely lacking the LPSA band. The LPS alterations resulted in changes in epitope exposure and thus, the receptor functions of the outer membrane as well as increased sensitivity of the bacterial outer membrane to lysis by human serum. In addition, alterations in phase variation frequency and sensitivity to several antibiotics were demonstrated as the result of the structural variations in LPS.

\section{METHODS}

Strains and mAbs. Strains used in this study were $B$. pertussis BP338 and BP347, obtained from A. Weiss (University of Cincinnati, OH, USA), and $B$. pertussis $\mathrm{MDH} 134+$, which was obtained from A. Wardlaw (Dept of Microbiology, University of Glasgow, UK) who originally received the strain from the Michigan Department of Health. BP347 is the avirulent Tn5 mutant which was produced by A. Weiss from BP338, which in turn was selected as a spontaneous nalidixic- acid-resistant mutant of Tohama 1. Likewise, MDH134- is the avirulent phenotype of MDH134 + which was selected by growth on Stainer-Scholte agar (Peppler, 1982). The LPS structure is conserved between virulent and avirulent species of the same strain. The Tn5-containing vector pUW964 was constructed and provided by A. Weiss (Weiss et al., 1983).

Bordetella cultures were maintained on Bordet-Gengou agar (BGA) with the appropriate antibiotics (Sigma) added. $B$. pertussis strain BP338 was maintained on BGA with $60 \mu \mathrm{g}$ nalidixic acid $\mathrm{ml}^{-1}$ while $\mathrm{Tn} 5$-insertion mutants were plated to BGA containing $60 \mu \mathrm{g}$ nalidixic acid $\mathrm{ml}^{-1}$ and $25 \mu \mathrm{g}$ kanamycin $\mathrm{ml}^{-1}$. Escherichia coli strain HB101 containing the Tn5 plasmid, pUW964, was maintained on L-agar containing $25 \mu \mathrm{g}$ kanamycin $\mathrm{ml}^{-1}$.

LPSA- and LPSB-specific mAbs, BL-2 and BL-8, respectively, were characterized previously by Archambault et al. (1991) and Martin et al. (1992). Ascites fluid containing the mAbs was stored at $-20^{\circ} \mathrm{C}$, then thawed and diluted in blocking buffer [3\% BSA in $50 \mathrm{mM}$ Tris-buffered saline, pH 7.0 (TBS)] for experiments.

Mutation of the wild-type B. pertussis genome by random insertion of a Tn5 transposon. B. pertussis strain BP338 was mutated by random insertion of a Tn5 transposon. The transposon was transferred though conjugation of the parental BP338 strain with an HB101 E. coli strain containing a Tn5 suicide vector, pUW964 (Weiss et al., 1983). B. pertussis strain BP338 was grown for $3 \mathrm{~d}$ on BGA containing $60 \mu \mathrm{g}$ nalidixic acid $\mathrm{ml}^{-1}$. E. coli strain HB101 containing the pUW964 suicide plasmid was grown overnight on L-agar containing $25 \mu \mathrm{g}$ kanamycin $\mathrm{ml}^{-1}$. Each organism was swabbed from its growth plate (normal BGA) onto a mating plate (BGA containing either $10 \mathrm{mM} \mathrm{MgCl}$ or $10 \mathrm{mM} \mathrm{MgSO}$ ) and incubated at $37^{\circ} \mathrm{C}$ for $2 \cdot 5-3 \cdot 0 \mathrm{~h}$. After incubation the organisms were swabbed from the mating plate to $2.0 \mathrm{ml}$ Stainer-Scholte broth (SSB) (Stainer \& Scholte, 1971). Mutants were selected by plating $100 \mu \mathrm{l}$ aliquots of a $10^{-1}$ dilution of this suspension onto BGA plates containing both $25 \mu \mathrm{g}$ kanamycin $\mathrm{ml}^{-1}$ and $60 \mu \mathrm{g}$ nalidixic acid $\mathrm{ml}^{-1}$. This protocol routinely resulted in 100-300 kanamycin-resistant, nalidixic-acid-resistant colonies per plate.

Western colony blot analysis. LPS mutants were screened by Western colony blotting with the LPSB mAb BL-8. A round, sterile, $82.5 \mathrm{~mm}$ diameter, nitrocellulose disc (Bio-Rad) was soaked in TBS and pressed evenly onto each plate of mutants. The disc was then floated on chloroform to fix the organisms to the nitrocellulose. Unoccupied sites on the nitrocellulose were blocked by incubating the disc in blocking buffer [TBS with $3 \%$ BSA] and $1 \%$ Bacto skimmed milk powder (Difco) for $3 \mathrm{~h}$ at room temperature.

The primary antibody, BL-8, was added to the blocking buffer at a dilution of $1: 100$ and the blot was incubated for a further $2 \mathrm{~h}$. The disc was then processed though a series of six $15 \mathrm{~min}$ washes in TBS then incubated for $2 \mathrm{~h}$ in blocking buffer containing goat anti-mouse alkaline phosphatase conjugate (Sigma). After an additional series of six $15 \mathrm{~min}$ washes the blot was developed in nitroblue tetrazolium/5-bromo-4chloro-3-indolyl phosphate (NBT/BCIP) as detailed by the manufacturer (Bio-Rad). Colonies which reacted with the anti-LPSB mAb, BL-8, were selected from the original plates, subcultured, and their LPS profiles confirmed by SDS-PAGE, silver stain, Western blot and dot blot analysis.

SDSPAGE, silver stain, Western blot and dot blot analysis. LPS mobility on SDS-PAGE was assessed from B. pertussis whole-cell lysates by the method of Laemmli (1970) as previously described (Peppler, 1984). Briefly, bacteria grown 
for $3 \mathrm{~d}$ on BGA containing $25 \mu \mathrm{g}$ kanamycin $\mathrm{ml}^{-1}$ were suspended in $50 \mathrm{mM}$ Tris/43 mM sodium glutamate/90 mM sodium chloride ( $\mathrm{pH} 7.5$ ) (TGS) in $13 \times 100 \mathrm{~mm}$ screw-cap test tubes to an $\mathrm{OD}_{540}$ of 0.3 . The organisms were centrifuged for $10 \mathrm{~min}$ at $10000 \mathrm{~g}$, resuspended in $100 \mu \mathrm{l}$ Laemmli solubilization mixture and boiled for $5 \mathrm{~min}$ to disrupt the cells. Ten micrograms proteinase $\mathrm{K}$ was then added and the samples were incubated at $60^{\circ} \mathrm{C}$ for $2 \mathrm{~h}$ with intermittent vortexing. After cooling to room temperature, the LPS was precipitated by addition of 9 vols acetone and incubation on ice for $1 \mathrm{~h}$. After another centrifugation at $10000 \mathrm{~g}$ for $10 \mathrm{~min}$, the pellet was resuspended in $150 \mu \mathrm{l} \mathrm{Laemmli}$ solubilization mixture and boiled for an additional $5 \mathrm{~min}$. Samples were stored at $4{ }^{\circ} \mathrm{C}$ until required.

The discontinuous system of Laemmli was used with a Trisglycine buffer system (Laemmli, 1970). A Hoeffer SE 600 series vertical slab gel unit was used to produce $14 \times 16 \mathrm{~cm}$ polyacrylamide gels. Stock acrylamide solution, containing a ratio of $30: 0 \cdot 8$ of acrylamide to $N, N^{\prime}$-methylenebisacrylamide, was used to make $5 \%$ total acrylamide for the stacking gel and $18 \%$ total acrylamide for the separating gel. Ten microlitres of each sample was applied to the wells and stacked at $5 \mathrm{~W}$ then separated at $15 \mathrm{~W}$ constant power with cooling to $4{ }^{\circ} \mathrm{C}$. Pre-stained low-molecular-mass markers from Gibco-BRL were used to follow the progress of each separation.

After proper separation had occurred (as indicated by the progress of the marker proteins and the dye front), the gels were either fixed in $40 \%$ ethanol and $5 \%$ acetic acid overnight, prior to silver stain, by the method of Tsai \& Frasch (1982) or transferred to $50 \mathrm{mM}$ sodium phosphate buffer $(\mathrm{pH} \mathrm{7.5)}$ and then assembled with a sheet of nitrocellulose slightly larger than the gel and loaded onto a Bio-Rad Trans-Blot apparatus according to the manufacturer's instructions. Transfer of the LPS from the gel to the nitrocellulose was completed after $3 \mathrm{~h}$ (as evidenced by pre-stained protein markers) utilizing $50 \mathrm{mM}$ sodium phosphate buffer under a constant voltage of $27 \mathrm{~V}(0.7-1.0 \mathrm{~A})$ with cooling to $4{ }^{\circ} \mathrm{C}$ (Peppler, 1984).

The nitrocellulose membrane was transferred to a polypropylene dish and incubated at room temperature for $3 \mathrm{~h}$ in $3 \%$ BSA in TBS. The primary antibody (mAbs BL-2 or BL-8) was added to the blocking buffer at a dilution of $1: 100$ and the blot was allowed to incubate for a further $2 \mathrm{~h}$ at room temperature. The membrane was then processed though a series of six 15 min washes in TBS after which it was incubated for $2 \mathrm{~h}$ in $3 \%$ blocking buffer containing secondary goat antimouse antibodies conjugated with alkaline phosphatase (Sigma) at a dilution of 1:5000. The blots were then developed with NBT/BCIP as described above for Western colony blots.

Dot blot analysis of the strains was performed using a Bio-Rad Bio-Dot apparatus. One hundred microlitres of an $\mathrm{OD}_{540} 0.3$ suspension in $13 \times 100 \mathrm{~mm}$ tubes of each strain was applied, per well, to nitrocellulose. The dot blots were incubated with primary then secondary antibodies and developed with NBT/BCIP as described above for Western blots.

Antibody accessibility radioimmunobinding assay. The protocol followed was detailed by Martin et al. (1992). Briefly, $5 \times 10^{9}$ c.f.u. $\mathrm{ml}^{-1}$ of 2 -d-old cultures of $B$. pertussis were incubated with the $\mathrm{mAb}(\mathrm{BL}-8$ or $\mathrm{BL}-2)$ and $0.25 \mu \mathrm{Ci}(9.25 \mathrm{kBq})$ ${ }^{125} \mathrm{I}$-labelled goat anti-mouse $\mathrm{IgG}$. The quantity of $\mathrm{mAb}$ bound to each strain of bacteria was measured with a gamma counter and expressed as counts per min per sample.

Preparation of Tn5 probes. Probes were prepared by labelling a complete $\operatorname{Tn} 5$ transposon using a random primer insertion method and two different labelling agents (Sambrook et al., 1989). A nonradioactive Tn5 probe was created using digoxigenin as the labelling agent (Boehringer Mannheim). Also, ${ }^{32} \mathrm{P}$ (Amersham) was used to radioactively label Tn5. Each probe was similarly reactive for our purposes.

Southern blot analysis. Genomic DNA was isolated by the CTAB method (Ausubel et al., 1987). DNA was restricted for $2 \mathrm{~h}$ at $37^{\circ} \mathrm{C}$ with five separate restriction enzymes: BamHI, ClaI, EcoRI, SalI and SmaI. Digests were run on $0.6 \%$ agarose gels at $100 \mathrm{~V}$ for $3 \mathrm{~h}$. Southern analysis was carried out as detailed in Sambrook et al. (1989) and Southern (1975). Blots on Hybond-N membrane were probed and developed with the digoxigenin (DIG) system (Sambrook et al., 1989) or with ${ }^{32} \mathrm{P}$ labelled Tn5 probes which were produced using the random primer labelling kit from Gibco-BRL.

One bacterial suspension of each strain was used to make preparations of LPS and genomic DNA (in agarose blocks for pulsed-field gel analysis and free in solution for Southern analysis) for each strain. SDS-PAGE, standard Southern analysis and PFGE Southern analysis were performed concurrently for each strain.

PFGE analysis. Control organisms (BP338, BP347) and all ten mutants (MLT1-10) were analysed by PFGE using the restriction enzymes $X b a \mathrm{I}$ and $S p e \mathrm{I}$ and following the methods of de Moissac et al. (1993). The DNA bands were sized by comparison of migration distances to those of the $\lambda$ DNA ladder standards (New England Biolabs). The position of the Tn5 inserts on the B. pertussis genome map created by Stibitz \& Garletts (1992) was determined by comparison of the XbaI and SpeI fragments with those of the published PFGE restriction map (Stibitz \& Garletts, 1992). To determine which pulsed-field bands contained the Tn 5 insertion, Southern hybridizations were done with a DIG-labelled Tn5 probe. The pulsed-field gel was stained with ethidium bromide and photographed followed by depurination in $0.25 \mathrm{M} \mathrm{HCl}$ for $15 \mathrm{~min}$ to ensure adequate transfer of the DNA to the nylon membrane. The DNA was transferred to Hybond-N nylon membrane (Amersham) over $24-36 \mathrm{~h}$ by capillary transfer using the method of Southern (1975) (Sambrook et al., 1989).

The washed blot was then placed in blocking buffer [digoxigenin blocking reagent (Boehringer Mannheim)] and 10\% foetal calf serum in maleate buffer $(100 \mathrm{mM}$ maleic acid, $150 \mathrm{mM} \mathrm{NaCl}, \mathrm{pH} \mathrm{7.5)}$ for $5 \mathrm{~h}$ at room temperature after which the anti-DIG antibody-alkaline phosphatase conjugate (Boehringer Mannheim) was added at a dilution of 1:10000 for a further incubation of $2-3 \mathrm{~h}$.

A series of six 30 min washes in maleate buffer preceded the addition of the LumigenPPD detection solution (Boehringer Mannheim). The blot was removed from the LumigenPPD solution, wrapped in plastic wrap, and incubated at $37^{\circ} \mathrm{C}$ for $15 \mathrm{~min}$. It was then exposed to X-OMAT AR autoradiograph film (Kodak) for 1-3 h.

The developed film was compared with full size photos of the ethidium-bromide-stained gel as well as films of the blot probed with the DIG-Tn 5 probe at a less stringent hybridization temperature $\left(37^{\circ} \mathrm{C}\right)$. The chomosomal position of the Tn5 insert was determined from analysis of and comparison with the $\mathrm{XbaI} /$ SpeI pulsed-field maps of Stibitz \& Garletts (1992).

Bactericidal assay with mAbs. Mutants and control organisms were suspended in sterile TGS to an $O_{540}$ of $0.17-0.20$ as above, and $50 \mu \mathrm{l}$ each suspension was transferred to a 96-well plate. The primary antibody (either BL-8 or BL-2) was heated for $30 \mathrm{~min}$ at $56^{\circ} \mathrm{C}$ to destroy any residual complement 
activity in the ascites fluid. The antibody was then serially diluted tenfold from $1 \times 10^{-1}$ to $1 \times 10^{-6}$ in PBS $(0.073 \mathrm{M} \mathrm{NaCl}$, $0.013 \mathrm{M} \mathrm{KH}_{2} \mathrm{PO}_{4}, 0.054 \mathrm{M} \mathrm{Na}_{2} \mathrm{HPO}_{4}, \mathrm{pH} \mathrm{7.35)}$ and $25 \mu \mathrm{l}$ each dilution was added, in triplicate, to wells containing the bacterial suspensions. The organisms were allowed to incubate with the antibody for $1 \mathrm{~h}$ at $37^{\circ} \mathrm{C}$.

Normal guinea pig serum was utilized as a source of complement. The amount of serum causing $50 \%$ haemolysis $\left(\mathrm{CH}_{50}\right)$ was determined using sheep red blood cells sensitized with haemolysin (Cedar Lane) so that a constant amount of complement activity could be used from one experiment to the next. Twenty-five microlitres of a 1:16 dilution (two $\mathrm{CH}_{50}$ units) of the guinea pig serum was added to the antibody and bacteria suspension and the mixture was allowed to incubate at $37^{\circ} \mathrm{C}$ for another hour. Ten millilitres of the total suspension was then put though a series of six fivefold serial dilutions. Ten microlitres of each dilution was then plated onto BGA and colonies counted after $3-4 \mathrm{~d}$.

Complement fixation. After plating the samples for the bactericidal test, the remaining $90 \mu \mathrm{l}$ from the bacteria, antibody, and complement suspension was incubated with $90 \mu \mathrm{l}$ of a $3 \%$ solution of sensitized sheep red blood cells (sRBCs) in PBS and incubated for $1 \mathrm{~h}$ at $37^{\circ} \mathrm{C}$. The cells were collected by centrifugation for $10 \mathrm{~min}$ at $500 \mathrm{~g}$ and the supernatant was transferred to a flat-bottomed 96-well ELISA plate. The extent of complement fixation was determined by measuring the amount of haemolysis after incubation of the various bacterium/antibody combinations measured at $\mathrm{OD}_{\mathbf{5 4 0}}$. Results from mutant strains were compared with negative controls which contained no bacteria or no added $\mathrm{mAb}$. The positive control used was the combination of Bordetella parapertussis 17903 with the $B$. parapertussis-specific mAb D13B11 which produced $100 \%$ complement fixation and $100 \%$ bactericidal activity. The extent of complement fixation was measured as the total complement available $(100 \%)$ minus the percentage of complement which remained free (after incubation with the mAb and bacteria) to lyse sRBCs.

Antibiotic sensitivity assay. Strains were grown for $2 \mathrm{~d}$ on BGA then suspended in $4 \mathrm{ml} \mathrm{SSB}$ to an $\mathrm{OD}_{540}$ of $0 \cdot 20$ as before. The suspension was then diluted and plated in duplicate to BGA plates to determine the total viable count of organisms used in the inoculum. The agents were selected based on previous studies on the interaction of various antibiotics with Bordetella species and enteric endotoxin (Bannatyne \& Cheung, 1984; Bass et al., 1969; Roantree et al., 1977; Sanderson et al., 1974; Peppler \& Schrumpf, 1984).

Twofold serial dilutions of each antibiotic were performed in duplicate in 96-well tissue culture plates from a stock solution which was predetermined using the parental strain, BP338, as a reference. Control wells with the antibiotic diluent alone were included in each assay. One hundred millilitres of a $10^{-1}$ dilution of bacteria was added to the final $100 \mathrm{ml}$ volume of each antibiotic or control (diluent, alone) well. The plates were incubated for $4 \mathrm{~d}$ at $37^{\circ} \mathrm{C}$. The MIC was taken as the last clear well (no turbidity) both visually and by ELISA reader at $\mathrm{OD}_{540}$. Final MIC data were based on geometric mean titres.

Phase variation assay. The rate of phase variation was measured by differentiating virulent from avirulent organisms using haemolysis, colony morphology and erythromycin resistance (Peppler, 1984; Weiss et al., 1983). Virulent organisms are documented to be domed, haemolytic and erythromycin-sensitive. Avirulent organisms are flat, nonhaemolytic and less erythromycin-sensitive. Bacteria were grown at $37^{\circ} \mathrm{C}$ for $2 \mathrm{~d}$ on BGA then suspended in SSB to an OD of 0.2 as above. Dilutions of $10^{-4}, 10^{-5}$ and $10^{-6}$ were made in SSB and $100 \mu \mathrm{l}$ of each was plated in triplicate onto plates of BGA alone, and BGA with $0.25 \mu \mathrm{g}$ erythromycin $\mathrm{ml}^{-1}$. Plates were incubated for $3 \mathrm{~d}$ at $37^{\circ} \mathrm{C}$. BGA plates were counted and colonies differentiated on the basis of haemolysis and erythromycin sensitivity. The number of variant colonies (nonhaemolytic or erythromycin-resistant) was divided by the total colony number on plain BGA to give the frequency of variation.

\section{RESULTS}

\section{General characteristics of LPS mutants}

All of the LPS mutant strains obtained by Tn5 insertion demonstrated a colonial morphology which is phenotypically identical to the parent strain, BP338. That is, all mutant strain colonies were domed and haemolytic on BGA. Growth rate, likewise, was unaffected by the presence of the $\operatorname{Tn} 5$ insert. The mutant colonies like the parental strain, BP338, all formed colonies of approximately $1 \mathrm{~mm}$ in diameter in approximately $3 \mathrm{~d}$. As well, the presence of haemolytic activity indicated a retention of parental virulence, although three mutants, MLT2, MLT3 and MLT5, produced avirulent phase variants at a greater frequency than did the other seven mutants or the parent strain (see below).

All potential LPS mutants were selected using the BL-8 LPSB-specific mAb. BP338 does not react with BL-8 due possibly to a masking of the LPSB by LPSA or the low amount of LPSB present in the BP338 outer membrane. A positive reaction of $\operatorname{Tn} 5$ mutant BP338 colonies (nalidixic-acid- and kanamycin-resistant) with BL-8 indicated that some alteration had occurred in the bacteria which either made the LPSB more accessible or significantly increased the amount of LPSB present. Coomassie-Blue- and silver-stained SDS-PAGE gels of whole-cell lysates indicated no visible alteration in protein content in any of the selected mutants. Of the hundreds of kanamycin-resistant mutant colonies assayed, ten mutants were found which reacted positively with the BL-8 mAb.

\section{Analysis of phenotypic alterations in the profiles of the isolated LPS of Tn5 mutants}

Mobility in SDSPAGE. The ten mutants that reacted with $\mathrm{mAb}$ BL-8 were further characterized by differences in mobility on silver-stained SDS-PAGE gels (Fig. 1).

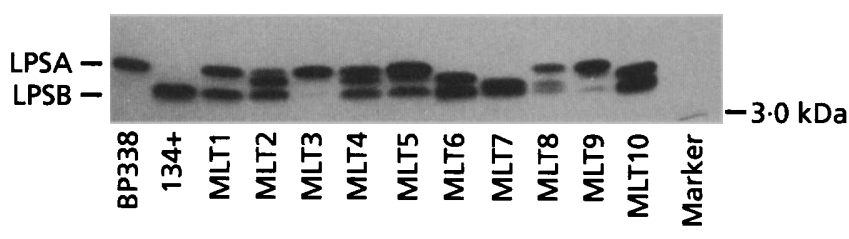

Fig. 1. Silver-stained SDS-PAGE profile of LPS from proteinase$K$-treated Laemmli digests of $B$. pertussis standard strains and Tn5-insert LPS mutants MLT1-10. 
Mutant strains MLT3, MLT5, MLT8 and MLT9 possessed abundant LPSA SDS-PAGE profiles similar to that of BP338 while MLT6, MLT7 and MLT10 resembled MDH134+ in its possession of more pronounced quantities of LPSB. Mutants MLT1, MLT2 and MLT4 had notable quantities of both LPSA and LPSB and thus, phenotypically resembled both BP338 and $\mathrm{MDH} 134+$. Based on mobility of pre-stained molecular mass markers, $B$. pertussis LPS band mobility (LPSA, LPSB and intermediates) indicated an apparent molecular mass in the range $4 \cdot 5-5 \cdot 5 \mathrm{kDa}$.

In several mutants, the LPS profiles comprised up to four specific molecular LPS types (Fig. 1). Between the bands commonly accepted as LPSA and LPSB lay two intermediate species which we have designated IntA and Int $B$ (for intermediate $A$, the band closest in apparent molecular size or mobility to LPSA and intermediate B, the species closest to LPSB). Where the control LPS, BP338 and MDH134 +, completely lacked these bands, the mutants all contained varying amounts of these intermediate species.

BP338 does possess a well-documented LPSB band which, though minor, was clearly evident on a more heavily loaded silver-stained SDS-PAGE gel (Martin $e t$ al., 1992; Peppler, 1984). Unfortunately, these measures seriously decreased the clarity of the four individual LPS bands, LPSA, LPSB, IntA and IntB, and this band is therefore not shown on this gel.

Only one mutant, MLT3, was nearly identical to the original parental phenotype, BP338. MLT3, however, demonstrated an increased reactivity with the LPSB $\mathrm{mAb}, \mathrm{BL}-8$, on Western colony blots. MLT5, MLT8 and MLT9, though possessing a dominant LPSA band, also contained considerable quantities of LPSB and IntA.

MLT1, MLT2 and MLT4 all contained significant amounts of both LPSA and LPSB bands but it was evident that these strains vary in the number and proportion of their LPS molecular species, with MLT2 and MLT4 containing IntA, as well as different concentrations of LPSA and LPSB.

Based on analysis of mobility on SDS-PAGE, the LPS profile of MLT7 was the most comparable to the LPS profile exhibited by the LPSB reference strain MDH134. However, the profile indicated the addition of an IntB band as well as the consistent presence of a very faint higher molecular mass band (approx. $7 \mathrm{kDa}$ ) of unknown composition. This band has been postulated to be an LPS aggregate or proteinase-K-resistant protein contaminant. MLT6 and MLT10 appeared identical to each other on SDS-PAGE. As well, both had IntB bands which are nearly equal in amount to their concentration of LPSB, thus distinguishing them from the standard MDH134 LPSB phenotype. Overall, we obtained nine LPS mutant phenotypes which differ significantly from the only clinically recognized $B$. pertussis LPS phenotypes, LPSAB (BP338) and LPSB (MDH134).

Western blot analysis. Though all mutant LPS strains were initially selected by reactivity of whole organisms (a)
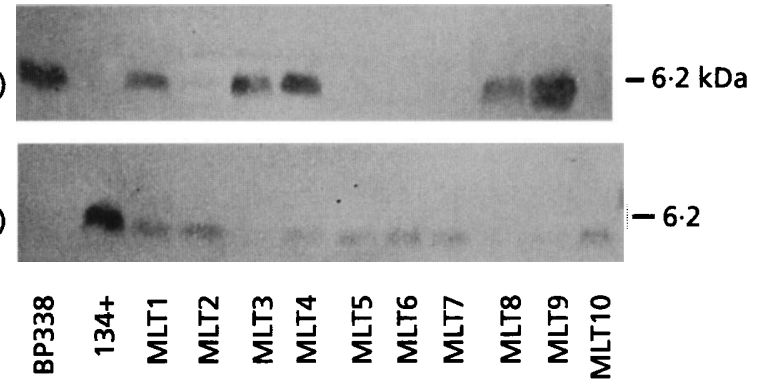

Fig. 2. Western immunoblot assay of reactivity of LPS-specific mAbs with SDS-PAGE of proteinase-K-treated, Laemmlisolubilized whole cells of $B$. pertussis standard strains and Tn5insert LPS mutants MLT1-10. Binding of (a) LPSA-specific mAb $B L-2$ and (b) LPSB-specific mAb BL-8.

with the BL-8 anti-LPSB mAb via colony blot, further examination using three additional immunoassays indicated significant differences between the mutants.

Western immunoblotting qualitatively assessed the relative reactivity of isolated LPS (by Laemmli solubilization and proteinase $\mathrm{K}$ digestion) of the various mutant and standard strains with mAbs specific for LPSA (Fig. 2a) and LPSB (Fig. 2b). In consideration of the subjective nature of this assay several Western blots were run on the mutants with minor variations in the standard assay conditions, most notably development time.

The data in Table 1 are means from five Western blots which were varied slightly in development time, blocking buffer, antibody dilution, etc., as a means of determining the most accurate account of each mutant's reactivity with the LPSA and LPSB mAbs. As such, there are minor differences between the overall averaged data contained in Table 1 and Fig. 2, notably the decreased reactivity of MLT4 and MLT5 to BL-8 as presented in Table 1. The averaged blot results serve as a point of comparison for the reactivity of the isolated LPS of the various mutants in Western blot analysis with the reactivity of the LPS as presented on intact bacteria (dot immunoassay, accessibility data and complement fixation).

Generally, Western blot results correlated well with SDS-PAGE profiles. If comparably large amounts of LPSA or IntA were present in the SDS-PAGE profile then the mutant reacted strongly with BL-2. If large LPSB or IntB bands were evident then the isolated LPS reacted more strongly with BL-8. For example, the MLT4 reacted less strongly than MLT2 with BL-8. This concurred with SDS-PAGE analysis where more LPSA could be seen in MLT4 than in MLT2 (Table 1, Fig. 1).

Similarly, mutants MLT3, 4, 8 and 9 bound more strongly with the LPSA mAb BL-2, as could be expected from the large LPSA and IntA bands which were present in their SDS-PAGE profiles. Mutants whose SDS-PAGE patterns showed major LPSB and IntB bands such as MLT6, 7 and 10 were more reactive with the LPSB mAb, BL-8. The presence of both LPSA and LPSB in large 
Table 1. Reactivity of LPSA-specific mAb BL-2 and LPSB-specific mAb BL-8 to B. pertussis standard strains and LPS mutant strains MLT1-10

Western blot and dot blot results are graded by colour intensity: ++++ , very strong reaction; +++ , strong reaction; ++ , moderate reaction; + , slight reaction; - , no reaction; $+/-$, reactivity which is clearly visible upon extreme development of the blot in substrate buffer.

\begin{tabular}{|c|c|c|c|c|c|}
\hline \multirow[t]{2}{*}{$\begin{array}{l}\text { SDS-PAGE } \\
\text { LPS type }\end{array}$} & \multirow[t]{2}{*}{ Strain } & \multicolumn{2}{|c|}{$\begin{array}{l}\text { Isolated LPS } \\
\text { (Western blot) }\end{array}$} & \multicolumn{2}{|c|}{$\begin{array}{l}\text { Intact bacterial cell } \\
\text { assay (dot blot) }\end{array}$} \\
\hline & & $\begin{array}{c}\text { BL-2 } \\
\text { (LPSA) }\end{array}$ & $\begin{array}{c}\text { BL-8 } \\
(\text { LPSB })\end{array}$ & $\begin{array}{c}\text { BL-2 } \\
\text { (LPSA) }\end{array}$ & $\begin{array}{c}\text { BL-8 } \\
\text { (LPSB) }\end{array}$ \\
\hline \multicolumn{6}{|l|}{$A, B$} \\
\hline$(A \ggg B)$ & BP347 & ++++ & + & +++ & - \\
\hline$(A \gg B)$ & BP338 & ++++ & - & +++ & - \\
\hline$(A \ggg B)$ & Tohama 1 & ND & ND & ND & ND \\
\hline$(A \gg B)$ & MLT3 & +++ & $+/-$ & +++ & - \\
\hline$(\mathrm{A}=\mathrm{B})$ & MLT1 & +++ & +++ & +++ & +++ \\
\hline \multicolumn{6}{|l|}{$A, B$, Int A } \\
\hline$(\mathrm{B}, \operatorname{Int} \mathrm{A} \gg \mathrm{A})$ & MLT2 & + & +++ & +++ & + \\
\hline$(B=\operatorname{Int} A=A)$ & MLT4 & +++ & + & +++ & - \\
\hline$(A>B>\operatorname{Int} A)$ & MLT5 & ++ & + & +++ & - \\
\hline$(A \gg B$, Int $A)$ & MLT9 & +++ & + & +++ & + \\
\hline \multicolumn{6}{|l|}{ A,B,IntB } \\
\hline \multicolumn{6}{|l|}{ B } \\
\hline (B) & MDH134- & - & ++++ & - & +++ \\
\hline (B) & MDH134+ & - & ++++ & - & +++ \\
\hline \multicolumn{6}{|l|}{ B,Int A } \\
\hline$(\operatorname{Int} A=B)$ & MLT6 & - & +++ & - & +++ \\
\hline$(\operatorname{Int} A=B)$ & MLT10 & - & +++ & - & +++ \\
\hline $\begin{array}{l}\text { B,IntB } \\
(\operatorname{IntB}=B)\end{array}$ & MLT7 & - & ++ & - & +++ \\
\hline
\end{tabular}

amounts, as was indicated by the SDS-PAGE profile of MLT1, was confirmed by its strong reaction to both mAbs.

\section{Dot blot and radioimmunoassay analysis of alteration in LPS as presented in the intact cell}

The dot immunoassay (Table 1 ) and the ${ }^{125}$ I radioimmunobinding assay (Fig. 3a) measured the ability of the LPSA- and LPSB-specific mAbs to bind to the LPS as presented in intact organisms. The two assays showed agreement within each strain and were, for the most part, consistent with the SDS-PAGE and Western blot profiles. For example, MLT1 had almost equal amounts of LPSA and LPSB on SDS-PAGE and reacted equally well with both BL-8 and BL-2 in the whole-cell assays. Mutants MLT2, MLT4 and MLT5, however, were exceptions in that these mutants showed some variation in their overall reactivity to the LPSA and LPSB monoclonals in the whole-cell assays. Isolated MLT2 LPS reacted better with BL- 8 whereas intact MLT2 reacted better with BL-2. MLT4 and MLT5 reacted strongly with BL-8 in the ${ }^{125}$ I-labelled $\mathrm{mAb}$ radioimmunoassay only.
Also, MLT2 and MLT5 reacted far less strongly with the LPSA monoclonal, BL-2, in the Western blot than they did in the other whole-cell immunoassays. The isolated LPS in the Western blot analysis of these two mutants likewise appeared to react differently to the LPSB-specific mAb, BL-8, in this case better, than would have been expected from the whole-cell immunoassays. These discrepancies are possibly due to the presence of intermediate LPS species (IntA and IntB) and the difference in species proportion as indicated in the SDSPAGE profile. Another explanation could be a mutationrelated change in surface exposure of the BL-2 and BL8 epitopes. Other than these discrepancies, results from the two immunoassays correlated well with data from SDS-PAGE analysis. For this reason, Table 1 is organized on the basis of SDS-PAGE LPS type for reference and comparison.

\section{Genetic analysis of Tn5 location in genomic DNA of LPS mutant strains}

Southern blot analysis. The presence of a single $\operatorname{Tn} 5$ insert in each of the mutant strains was confirmed by Southern analysis (Fig. 4). The Tn5 probe hybridized with a single 

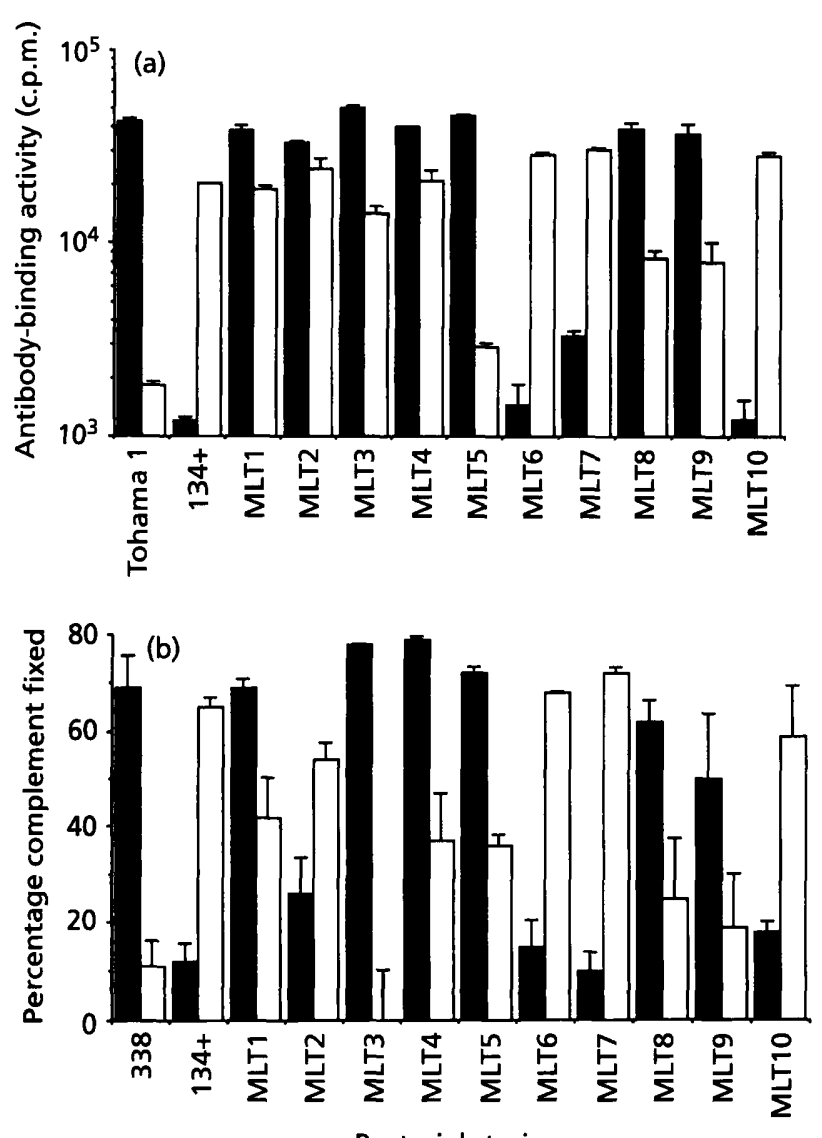

Fig. 3. (a) ${ }^{125} /$-radioimmunobinding assay of accessibility of whole $B$. pertussis mutant and standard strains to LPSA- and LPSBspecific $m A b s, B L-2$ and BL-8, respectively. Standard deviations of the means of three independent assays are indicated. (b) Complement-fixing ability of $B$. pertussis LPS mutant and standard organisms in conjunction with mAbs BL-2 and BL-8, respectively. Haemolysis of sensitized red blood cells (sRBCs) was measured spectrophotometrically by analysis of released oxyhaemoglobin at an $O D_{540}$. The extent of complement fixation was measured as the total complement available (100\%) minus the percentage of complement which remained free (after incubation with the mAb and bacteria) to lyse sRBCs. Standard deviations of the means of three independent assays done in quadruplicate are indicated. Solid bars, BL-2; open bars, BL-8.

band in mutant and control strains when DNA was digested with enzymes such as ClaI and EcoRI which do not recognize sites within the $\operatorname{Tn} 5$ gene. When digested with enzymes such as BamHI, SmaI and SalI which have a single restriction site within the Tn5 gene each DNA digest produced two bands which hybridized with the Tn5 probe. The lack of two distinct bands with digests of BP347 is likely due to a similarity in size of the two restriction fragments and the poor resolution of small differences in such large fragments when using a $0.6 \%$ agarose gel system.

Restriction digests of MLT5 and MLT6 with all five enzymes yielded Tn5 hybridized bands of similar sizes. This was also the case for digests of MLT7 and MLT8. This similarity in band size for five different restriction
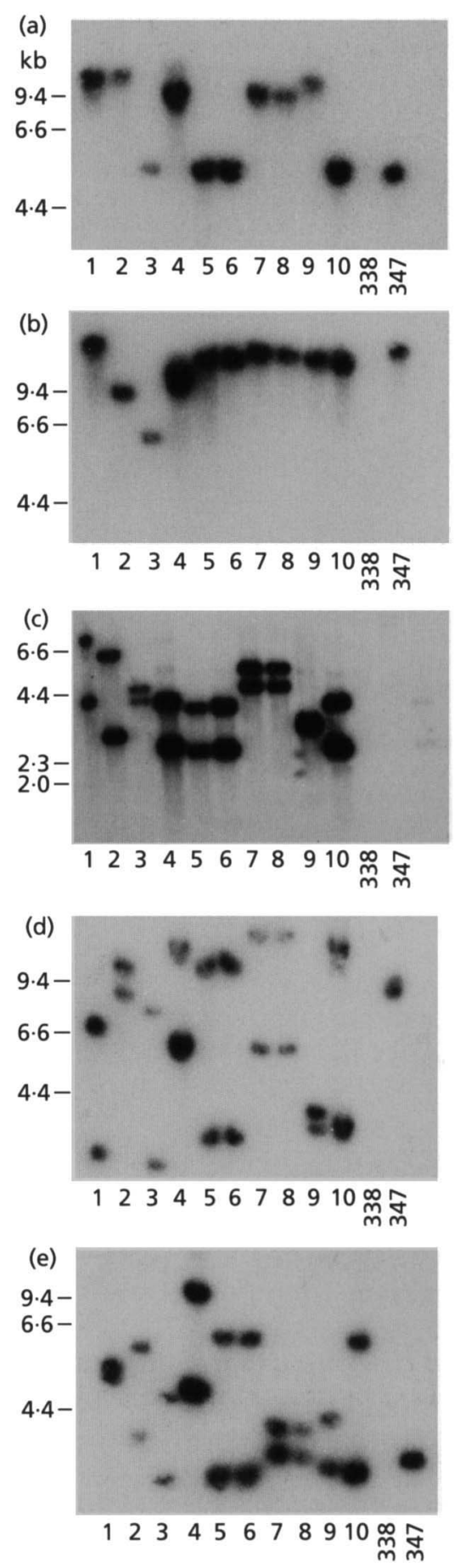

Fig. 4. Autoradiographs of Southern blot analysis of band location of ${ }^{32} \mathrm{p}$-labelled Tn5 probe in $B$. pertussis LPS mutants restricted with (a) EcoRI, (b) Clal, (c) Smal, (d) BamHI and (e) Sall. Molecular mass markers are shown on the left.

enzymes indicates a similarity between the structure of the genes in which the Tn5 is inserted in MLT5 to that in MLT6 and the gene of MLT7 to that in MLT8. However, in subsequent Southerns with better-resolved fragments, the mobilities of the pairs of bands were 


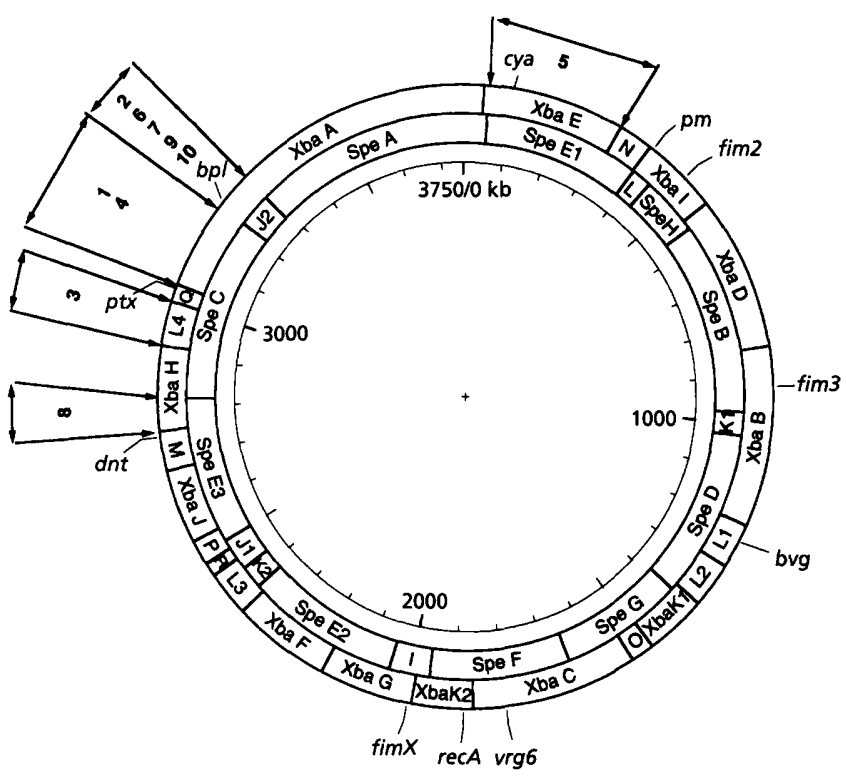

Fig. 5. Location on B. pertussis genomic map of the Tn5 inserts in LPS mutants MLT1-10 (numbers inside arrows) and for reference, genes for $B$. pertussis LPS $(b p /)$, adenylate cyclase (cya), pertactin (prn), fimbriae $X, 2$ and 3 (fimX, fim 2 and fim 3 , respectively) Bordetella virulence genes (bvg), vir-repressed gene 6 (vrg6), recA, dermonecrotic toxin (dnt), and pertussis toxin (ptx) (Stibitz \& Garletts, 1992).

clearly different (data not shown). Also, the pulsed-field data below indicate that the Tn5 insert in MLT5 is not close to that of MLT6 on the BP338 chromosome; the same is true for the Tn5 inserts in strains MLT7 and MLT8.

PFGE analysis. Agarose blocks of the genomic DNA of mutant strains were restricted with $X b a \mathrm{I}$ and $S p e \mathrm{I}$ then analysed by PFGE. Incubation of the blotted pulsed-field gels with a DIG-labelled Tn5 probe yielded the approximate genomic location of each $\operatorname{Tn} 5$ insertion (Fig. 5).

As all mutants were derived from BP338, we were able to use the pulsed-field gel map created by Stibitz \& Garletts (1992) to locate the disrupted genes on the B. pertussis genome using a $\operatorname{Tn} 5$ probe (Table 2). By coordinating the band location of the Tn5 in XbaI digests of the mutants with the Tn5 location in a SpeI digest of the mutants, the general location of the genes responsible for the LPS phenotypic changes was determined. Overall, the Tn5 was located in five different regions for the ten mutants based on $\mathrm{XbaI}$ and SpeI fragment positions.

Tn5 insertions in strains MLT2, MLT6, MLT7, MLT9 and MLT10 all mapped to an approximately $100 \mathrm{~kb}$ region at approximately $3200-3300 \mathrm{~kb}$ on a $3750 \mathrm{~kb}$ map of the B. pertussis chomosome (XbaI fragment A and SpeI fragment J2). MLT1 and MLT4 map to the area immediately upstream of this region at approximately $3000-3200 \mathrm{~kb}$ in a $200 \mathrm{~kb}$ region (Xbal fragment $\mathrm{A}$ and Spel fragment C). MLT3 is located in another approximately $100 \mathrm{~kb}$ region between $2900-3000 \mathrm{~kb}$ along the
Table 2. PFGE and mapping of Tn5 insertions in LPS mutants MLT1-10 to the parental $B$. pertussis strain (BP338) genome

Position of $\operatorname{Tn} 5$ insert on restriction-digested pulse-field gel as determined by $\mathrm{XbaI}$ and SpeI digest. Position of mutants on 3750 kb BP338 genome as mapped by Stibitz \& Garletts (1992).

\begin{tabular}{|c|c|c|c|c|}
\hline \multirow[t]{2}{*}{$\begin{array}{l}\text { SDS-PAGE } \\
\text { LPS type }\end{array}$} & \multirow[t]{2}{*}{ Strain } & \multicolumn{2}{|c|}{$\begin{array}{l}\text { Tn } 5 \text { band location } \\
\text { on PFGE map with: }\end{array}$} & \multirow[t]{2}{*}{$\begin{array}{c}\text { Position } \\
\text { (kb) }\end{array}$} \\
\hline & & $X b a I$ & SpeI & \\
\hline \multicolumn{5}{|l|}{$\mathrm{A}, \mathrm{B}$} \\
\hline & MLT1 & A & C & $3050-3200$ \\
\hline & MLT3 & L4 & $\mathrm{C}$ & $2950-3000$ \\
\hline \multicolumn{5}{|l|}{$A, B, I n t A$} \\
\hline & MLT2 & A & $\mathrm{J} 2$ & $3200-3300$ \\
\hline & MLT4 & A & $\mathrm{C}$ & $3050-3200$ \\
\hline & MLT5 & $\mathrm{E}$ & E1 & $60-300$ \\
\hline & MLT9 & A & $\mathrm{J} 2$ & $3200-3300$ \\
\hline \multicolumn{5}{|l|}{$\mathrm{A}, \mathrm{B}, \mathrm{IntB}$} \\
\hline & MLT8 & $\mathrm{H}$ & E3 & $2750-2820$ \\
\hline \multicolumn{5}{|l|}{$\mathrm{B}$, Int A } \\
\hline & MLT6 & A & $\mathrm{J} 2$ & $3200-3300$ \\
\hline \multirow{2}{*}{\multicolumn{5}{|c|}{$\mathrm{B}$, IntB }} \\
\hline & & & & \\
\hline & MLT7 & A & $\mathrm{J} 2$ & $3200-3300$ \\
\hline
\end{tabular}

map (XbaI fragment L4 and SpeI fragment C). The Tn5 insert in MLT8 maps to an $80 \mathrm{~kb}$ region slightly further from the main LPS gene cluster at approximately 2750-2820 kb (XbaI fragment $\mathrm{H}$ and SpeI fragment E3). MLT5 maps to a $240 \mathrm{~kb}$ genomic region located at approximately $60-300 \mathrm{~kb}$ from the origin (XbaI fragment $\mathrm{E}$ and Spel fragment E1).

\section{Analysis of alterations to bacterial outer membrane function}

Alterations in complement fixation and bactericidal activity of BL-2 and BL-8 mAbs in the presence of guinea pig complement. The basic pattern of reactivity in the complement fixation assay (Fig. 3b) was consistent with the radioimmunobinding accessibility data (Fig. 3a). For example MLT1 again reacted with both mAbs indicating the strong presence of both LPSA and LPSB in its structure. MLT3 fixed slightly less complement with the LPSB mAb, BL-8, than would have been expected from the accessibility data. The only major difference noted was the lower amount of complement fixed by MLT2 in the presence of BL-2 and BL- 8 than would have been expected from the accessibility data. This is not inconsistent, however, as Western blot analysis also indicated a dual mAb reactivity for MLT2 (reactivity with both LPSA and LPSB).

None of the mutant (MLT1 to MLT10) or control strains (BP338, BP347, MDH134+, MDH134-) was susceptible to the bactericidal activity of complement in 
Table 3. Alterations in antibiotic sensitivity of $B$. pertussis LPS mutant strains

All values are MICs in $\mathrm{mg} \mathrm{ml}^{-1}$. Nov, novobiocin; Ole, oleic acid; Sul, sulphamethoxazole; Tet, tetracycline; Tp, trimethoprim; Ami, amikacin; Kan, kanamycin; Str, streptomycin.

\begin{tabular}{|c|c|c|c|c|c|c|c|c|}
\hline \multirow[t]{2}{*}{ Strain } & \multirow{2}{*}{$\frac{\text { Increase }}{\text { Nov }}$} & \multicolumn{4}{|c|}{ Decrease } & \multicolumn{3}{|c|}{ Tn5-related decrease } \\
\hline & & Ole & Sul & Tet & $\mathrm{Tp}$ & Ami & Kan & Str \\
\hline \multicolumn{9}{|l|}{ Controls } \\
\hline BP338 & $0 \cdot 16$ & $0 \cdot 12$ & 4 & $0 \cdot 2$ & 4 & 16 & 8 & 15 \\
\hline BP347 & 0.03 & 1 & 0.25 & $1 \cdot 6$ & 16 & 96 & $>32$ & $>500$ \\
\hline $\begin{array}{l}\text { MDH134+ } \\
\text { Mutants }\end{array}$ & $0 \cdot 16$ & $0 \cdot 12$ & 0.5 & $0 \cdot 2$ & 1 & 8 & 4 & 5 \\
\hline MLT1 & 0.02 & $0 \cdot 25$ & 4 & $0 \cdot 8$ & 4 & 182 & $>32$ & $>500$ \\
\hline MLT2 & 0.02 & 0.50 & 16 & 0.6 & 16 & 144 & $>32$ & $>500$ \\
\hline MLT4 & 0.02 & 0.50 & 16 & 0.8 & 16 & 182 & $>32$ & $>500$ \\
\hline MLT7 & 0.03 & 0.50 & 16 & $0 \cdot 8$ & 24 & 182 & $>32$ & $>500$ \\
\hline MLT10 & $0 \cdot 16$ & $0 \cdot 25$ & 16 & $0 \cdot 8$ & 16 & 182 & $>32$ & $>500$ \\
\hline
\end{tabular}

the presence of either mAb (data not shown). Thus, the complex formed by the mAbs with complement was not capable of initiating bacterial lysis under the conditions used in this study.

Alterations in frequency of phase variation of LPS mutants. $B$. pertussis is capable of switching from virulent to avirulent phenotype under two distinct set of circumstances. Phase variation is the term given to the process by which approximately 1 in $10^{6}$ cells shifts to avirulence due to the occurence of a single characterized frameshift mutation. Antigenic modulation refers to the phenomenon in which a phenotypic shift of the entire culture occurs due to environmental stimuli.

During the course of these investigations significant differences were noted in the rate of phase variation of several of the LPS mutants. Standard passaging of cultures revealed a predisposition for loss of haemolytic activity in several of the mutants. The nonhaemolytic colonies did not revert to the haemolytic phenotype readily; thus the phenomenon was attributed to phase variation as opposed to antigenic modulation. When the actual rate of phase variation was measured it was found that MLT2, MLT3 and MLT5 all varied at higher rates than the parental strain. The phase variation frequency rates of these mutants were $2.6 \times 10^{-4}, 3.4 \times$ $10^{-4}$ and $1.5 \times 10^{-2}$, respectively. The frequency of variation for BP338 was $2.9 \times 10^{-6}$.

To confirm that the loss of haemolysis was indicative of a phase shift from virulence to avirulence as opposed to a disruption of the haemolytic activity alone, a second phase variation assay utilizing the heightened sensitivity of virulent organisms to erythromycin was used.

MLT5 produced no more erythromycin-resistant col- onies than did the virulent parental strain, BP338. The frequencies of colonies which grew on erythromycin as opposed to BGA alone were $3.1 \times 10^{-6}$ and $4.3 \times 10^{-6}$, respectively. MLT2 and MLT3, however, had frequencies of $1.7 \times 10^{-3}$ and $1.5 \times 10^{-4}$, respectively. The increases in erythromycin-resistant phase variants were consistent with the increased number of nonhaemolytic organisms although not equivalent to the frequency with which BP347 cells grew on erythromycin $(3.9 \times$ $10^{-1}$ ).

\begin{abstract}
Alterations in sensitivity of LPS mutants to antibiotics and outer-membrane-active agents. The sensitivity of the LPS mutants to numerous antibiotics and membrane-active reagents was assayed and compared to results obtained with the virulent parental strain BP338, its avirulent counterpart BP347, and the virulent LPSB strain MDH134 + (Table 3). Five strains were chosen from the original ten to provide a diverse range of LPS alterations; MLT1 (which contained equal amounts of LPSA and LPSB), MLT2 and MLT5 (which contained varied amounts of LPSA, IntA and LPSB), MLT10 (which contained only IntA and LPSB), and MLT7 (which contained only IntB and LPSB). In all instances total bacterial viability counts were between $3.0 \times 10^{7}$ and $2.0 \times 10^{8}$ c.f.u. $\mathrm{ml}^{-1}$. Only a difference in antibiotic sensitivity of two dilutions or more was considered a notable alteration in sensitivity (Table 3 ).
\end{abstract}

BP347 was significantly less sensitive to several antibiotics than was the virulent strain, BP338. Notable decreased sensitivity to amikacin and kanamycin was expected due to the kanamycin-resistance gene encoded by the $\operatorname{Tn} 5$ insert. The decreased sensitivity to streptomycin is not as clearly attributable to the transposonencoded kanamycin resistance but has been documented as an expected result of $\operatorname{Tn} 5$ insertion in previous studies (Weiss et al., 1983). BP347 also exhibited notable 
decreases in sensitivity to oxacillin, cefotaxime, cephalothin, erythromycin, fusidic acid, oleic acid, rifampicin, tetracycline and vancomycin. In comparison with BP338, BP347 exhibited notable increases in sensitivity to sulphamethoxazole and novobiocin. No notable difference was observed in the sensitivities of the control strains to the LPS-specific agents EDTA, EGTA and polymyxin B. MDH134 + displayed the same sensitivity as BP338 to erythromycin, novobiocin, oleic acid, tetracycline and EDTA. Notable increases in sensitivity were observed for sulphamethoxazole, trimethoprim and cefotaxime.

Most of the LPS mutant strains demonstrated notably decreased sensitivity to the hydrophilic antibiotics sulphamethoxazole and trimethoprim, while notable increased sensitivity to novobiocin was also documented. MLT1 was a conspicuous exception in its consistent similarity to the parental strain, BP338. There were no obvious differences in the sensitivities of MLT1 to erythromycin, fusidic acid, rifampicin, sulphamethoxazole, trimethoprim or vancomycin. As well, MLT2 showed no difference from BP338 in sensitivity to fusidic acid or cephalothin and MLT10 was similar to BP338 in its reaction to novobiocin.

\section{DISCUSSION}

Our goal in this study was to create and analyse a number of $B$. pertussis strains with genetically altered LPS which differed from the naturally existing LPSAB and LPSB phenotypes. By characterizing the properties of these specifically altered bacteria we hoped to clarify the role of LPS in outer membrane mechanics and, ultimately, B. pertussis infection and immunity. It is possible to modify the LPS to examine its effect on neighbouring molecules and the cell as a whole as was done in the creation of the 'rough' LPS chemotype mutants of Salmonella typhimurium (Liang-Takasaki et al., 1982; Osborne, 1968). Mutations resulting in progressively truncated LPS molecules produced significant changes in the pathogenicity of the organisms, such as increased sensitivity to complement-mediated killing as well as various antibiotics. The availability of a mAb specific for the mutant LPSB phenotype allowed us an efficient, specific screening of LPSB mutants which had been genetically 'tagged' with a $\operatorname{Tn} 5$ transposon (Martin et al., 1992).

This method of mutation and screening has produced ten Tn5 mutant strains derived from the wild-type LPSAB strain BP338 which react with the LPSB mAb, $B L 8$, to a much greater extent than the parental phenotype. Characterization by SDS-PAGE followed by silver staining of these mutants revealed two novel LPS bands, Int $A$ and IntB, in addition to the two commonly characterized LPS bands, LPSA and LPSB. Different banding (LPS) patterns were distributed over nine different LPS phenotypes (of the ten mutant strains tested, MLT6 and MLT10 appeared to be very similar in phenotypic and genetic characteristics and could possibly be the result of an insertion in the same gene).

The LPS mutants developed in our laboratory showed SDS-PAGE structural profiles which were consistent with observed alterations in their antigenic reactivity (epitope exposure). The LPSA, IntA-dominant phenotypes reacted well with BL-2 and more poorly with BL8 while the LPSB, IntB-dominant mutants reacted better with BL-8 than BL-2 (Table 1). There were only a few anomalies. MLT3, though almost identical to the parental strain BP338, was picked up by initial colony blots with BL-8 due to the increase in LPSB concentration which is clear on heavily developed Western blots and SDS-PAGE.

The only mutant to exhibit an overall inconsistency in its antigenic reactivity was MLT2. This mutant fixed a lesser amount of complement in the presence of BL-2 than would have been anticipated from accessibility data. Also, there was a noted difference in reactivity of the LPS monoclonals with cell-free LPS (Western blot) in comparison with the membrane-associated LPS found in intact bacterial cells (dot blot) (Table 1).

Some of these differences may be the result of differences between the presentation of isolated LPS separated then blotted from a gel and the presentation of LPS associated with the whole organism as is the case with the dot blot and accessibility data. IntA appears to be more reactive with the LPSA-specific BL-2 when presented in the whole cell, while simultaneously masking the LPSB epitope which reacts with BL-8. Upon disruption the LPSB is more exposed and the change in presentation or availability might make the IntA less similar to LPSA, drastically decreasing the reactivity of the BL-2. Differences in the IntA and LPSA proportions could explain the differences in reactivities among IntA-containing mutants. The reactivity of BL-2 appears to be exclusive to the LPSA band as shown by the lack of reactivity of phenotypes lacking this band such as MLT6 (IntB, LPSB) and MLT7 (IntB, LPSB). LPS phenotypes lacking LPSB were similarily unreactive with BL-8. This is in agreement with findings published recently by Allen $\&$ Maskell (1996) who genetically characterized a locus active in the biosynthesis of $B$. pertussis LPS. The LPS of bplG (which contains two bands analogous to Int A and LPSB) was unreactive with BL-2 as was an LPSB mutant bplH.

Analysis of the LPS mutants indicates that a major region responsible for genetic coordination of much of the observed phenotypic LPS heterogeneity is the area which stretches from approximately $2900-3300 \mathrm{~kb}$ on the $3750 \mathrm{~kb}$ genomic map, and encompasses genes responsible for MLT1, MLT2, MLT3, MLT4, MLT6, MLT7, MLT9 and MLT10. This region also contains the structural gene responsible for pertussis toxin production (Stibitz \& Garletts, 1992). The consequences of this genetic association, if any, are unknown.

PFGE mapping results indicated a clustering of the putative LPS genes in those mutants with phenotypic 
similarities. Four of the ten mutants, MLT2, MLT6, MLT7 and MLT10, which contained large amounts of LPSB or IntB and little, if any LPSA, were found to have genetic origins in a chomosomal region between 3200$3300 \mathrm{~kb}$. A second group (MLT1 and MLT4), which contained large amounts of both LPSA and LPSB, mapped to a similar region at $3050-3200 \mathrm{~kb}$ while those mutants whose LPS was composed mainly of LPSA (MLT3, MLT5, MLT8 and MLT9) had Tn5 inserts which were less localized on the chromosomal map.

Isolation and sequencing of the genes containing the transposon inserts will be an important documentation of the genetic organization of $B$. pertussis LPS biosynthesis. The $b p l$ locus characterized by Allen \& Maskell (1996) is in the SpeI J2 fragment where the Tn5 inserts of five of our mutants are found (MLT2, MLT6, MLT7, MLT9 and MLT10). In addition, the Tn5 inserts of MLT1 and MLT4 are just upstream of this region in a part of the SpeI C fragment. If Tn5 is inserted close to the $5^{\prime}$ end of this fragment, a polar effect of the transposon on genes in the $b p l$ locus may also be the cause of the LPS phenotype changes seen in these two mutants. Thus seven of our ten mutants may owe their LPS changes to alterations in the $b p l$ locus. But three of our Tn5 mutants, MLT3, MLT5 and MLT8, map in regions well outside the $b p l$ locus in XbaI L4, SpeI E1, and the $3^{\prime}$ end of $X b a \mathrm{I} \mathrm{H}$, respectively. The genetic basis for their LPS alterations may reveal other important genetic loci in LPS biosynthesis.

Southern analysis indicated a substantial amount of structural homology between the genes in which the Tn5 was inserted for two pairs of mutants: MLT5 and MLT6, and MLT7 and MLT8. Pulsed-field mapping did not place these mutants in a similar genomic location, however, strengthening the phenotypic evidence that each of the mutants in the pairs was the result of different gene disruptions.

A host of protein intermediates is responsible for assembly and transport of LPS. In S. typhimurium the biosynthesis of LPS has been demonstrated to be organized into discrete 'blocks' of genes (as termed by Makela \& Stocker, 1984) which coincide with the required function of a corresponding 'block' of gene products. For example, the genes required to synthesize the $\mathrm{O}$-unit are found in one cluster on the genome while another cluster responsible for core construction is found elsewhere. Separate blocks are believed to exist for construction of the primary components of the LPS (the O-unit, core and lipid A), the final assembly of the entire molecule, the addition of 'accessories' to the finished molecule and the regulation of the entire process. Additionally, LPS biosynthesis involves numerous 'housekeeping' genes for common precursor sugars, phosphorylation, etc., which are scattered around the genome.

As $70 \%$ of our mutants result from a disruption in the $250 \mathrm{~kb}$ area between 3050 and $3300 \mathrm{~kb}$ on the $3750 \mathrm{~kb}$ genome we postulate that the block of genes responsible for the assembly of the three-sugar 'O-antigen' of $B$. pertussis resides in this region, possibly as an operon. Whether the three sugars can be termed an O-unit and form a distinct block which is separate from that of the core sugars has yet to be determined. Allen \& Maskel (1996) present a convincing argument that the trisaccharide is indeed a small O-unit, truncation of which decreases the affinity of transfer to the core-lipid A construct in a manner similar to that documented in Shigella dysenteriae. Of the tens of thousands of colonies screened we failed to obtain any pure LPSB exclusive phenotypes as have been reported by other laboratories (i.e. MDH134, A100) (Caroff et al., 1990; Peppler, 1984). Why intermediate LPSs IntA and IntB were produced while the more clinically established LPSB phenotype was not is a matter of conjecture. An equally plausible scenario suggested by the unexpectedly large percentage of Int A,IntB mixed-band phenotypes in this study is that the three sugars are a part of the core and the result of deletions in different genes responsible for the sequential addition of these sugars. Thus, the assembly of the core may also be governed by the proposed gene 'block'. Further evaluation of the exact mechanism of $B$. pertussis LPS biosynthesis and possibly further mutation of the mutants we have created should shed some light on this issue.

It is probable that the mutants are the result of a disruption in genes encoding the construction or regulation of the sugars which comprise the proposed $\mathrm{O}$ antigen monomer which is found in LPSA but is lacking in LPSB (Lasfargues et al., 1993; Lebbar et al., 1994; Le Blay et al., 1996). Also, the variation in ratio of LPSA to LPSB (which is most visible in MLT1) presents the possibility of a regulatory as well as a structural role for some of the $\operatorname{Tn} 5$-altered genes. These proportional differences could be the result of a 'leaky' mutation or due to the alteration or deletion of any of a number of enzymes in, or associated with, the LPS biosynthetic pathway. The Tn 5 insert may also cause multiple polar effects if it inserted upstream of an operon (e.g. in a promoter). The cloning, sequencing and expression of the Tn5-tagged genes should help clarify the exact nature of the gene disruption responsible for the observed phenotypic changes.

Several studies have characterized the ability of Gramnegative endotoxin to activate complement and form the membrane attack complex which in turn leads to cell lysis (Archambault et al., 1991; Byrd et al., 1991; Fernandez \& Weiss, 1994). Thus, assays examining the ability of the variant strains to fix complement in the presence of the LPSA and LPSB mAbs served as initial attempts to characterize the consequences of LPS alterations on an important function of the outer membrane. Unlike physical assays such as SDS-PAGE analysis and Western blotting, the variation in complement fixation by the mutant LPS strains from the parental strain provided the first rudimentary correlation of structural LPS alteration with changes in bacterial interaction with the host. In organisms which have extensive $\mathrm{O}$-units, the loss of the most exposed sugars often leaves the cell vulnerable to complement- 
mediated killing. The effect on B. pertussis, which has a very truncated $\mathrm{O}$-chain, was different. Although the mutants were able to fix complement with the appropriate (i.e. reactive) $\mathrm{mAbs}$ the complex formed was unable to initiate membrane lysis under the experimental conditions utilized.

The effect of the LPS alterations on membrane barrier functions was further explored by analysing the effect of numerous antibiotics and cell membrane reactive agents on the mutants in comparison with control and parental strains. As the first erythromycin-resistant $B$. pertussis strain has recently been isolated the mechanism of interaction between various antibiotics and the outer membrane is of timely importance. All of the B. pertussis LPS mutant strains except MLT10 exhibited an $81-88 \%$ increase in sensitivity to the hydrophobic antibiotic novobiocin and a $300-400 \%$ decrease in sensitivity to the hydrophilic antibiotic tetracycline. These results are comparable to the most drastic differences documented in studies of the Salmonella typhimurium $\mathrm{Ra}-\mathrm{Re}$ LPS mutants which showed a $90-98 \%$ increase in sensitivity of the mutants to novobiocin and a 100-500\% decrease in sensitivity to tetracycline (Roantree et al., 1977; Sanderson et al., 1974). Organisms such as S. typhimurium are thought to become more sensitive to hydrophobic antibiotics as the result of the creation of 'patches' of phospholipid bilayer in an effort to compensate structurally for the loss of endotoxin-associated protein. A decrease in susceptibility to hydrophilic antibiotics is also linked to the loss of such porin transport proteins. Endotoxin-associated proteins are lost when an alteration in LPS structure destabilizes the noncovalent bonding of endotoxin to an adjacent molecule. This process occurs at precise structural points in both Salmonella (loss of terminal glucose from Rc mutant to produce $\mathrm{Rd}_{1}$ mutant) and $E$. coli (loss of phosphate groups on heptose in D21e7 mutant) (Nikaido \& Vaara, 1985). It must be noted that the relative structural truncation was far less drastic in the $B$. pertussis LPS mutants than the enteric species. Also, the chemical structure of $B$. pertussis LPS differs substantially from those endotoxins with naturally occurring extensive O-side chains. The LPS of $B$. pertussis, naturally bereft of an extensive O-unit, is electrostatically reinforced with divalent cations and a high degree of negative charges on its polysaccharide portion. The loss of even a small number of these charges could result in a noticeable change in the interactions between LPS molecules and between LPS and other molecules.

All mutant strains except MLT10 were notably less sensitive to the hydrophobic agent oleic acid. B. pertussis is distinct from many other Gram-negative organisms in its increased sensitivity to fatty acids such as oleic acid. This increased sensitivity is not fully understood but the data here suggest a role for the LPS in the bacterial sensitivity to fatty acids which so complicates the in vitro culture and isolation of $B$. pertussis. Notable decreases in sensitivity to the hydrophilic antibiotic sulphamethoxazole were also detected. As mentioned above, hydrophilic molecules pass though the Gramnegative outer membrane by way of protein porins. It is possible that the alterations in LPS structure have disrupted the conformation of associated porins, limiting their ability to transport molecules of a particular size, as was the case in several other studies (Nikaido \& Vaara, 1985). The consistent differences of the LPS mutants from the parental strain in sensitivity to antibiotics of varied chemical properties indicate a disruption in overall membrane integrity which was enough to allow alterations in the passage of hydrophilic as well as hydrophobic agents into the cell. Thus, changes in the membrane are the result of more than alterations in the gross structural barrier properties of the LPS polysaccharide moiety alone.

Phase variation is the phenomenon by which a shift from virulent to avirulent phenotype occurs due to a specific frameshift mutation. It involves a number of outer-membrane-associated receptors, factors and toxins. B. pertussis LPS, however, has never been associated with this process. As an unexpected result of the Tn5 mutation three of the LPS mutants were observed to undergo this phenomenon at a higher frequency than the parental strain. Two distinct characteristics of the phase variation shift from virulence to avirulence were used to confirm these increases: the lack of haemolysis which occurs upon shifting to an avirulent phenotype and the decrease in sensitivity to erythromycin. Haemolysis is controlled by adc, the adenylate cyclase toxin-haemolysin gene, which in turn is controlled by a trans-acting vir operon during phase variation. Three mutant strains demonstrated an elevated frequency of shifts from haemolytic (virulent) to nonhaemolytic (avirulent) phenotypes. To differentiate between disruptions in the phase variation phenomenon and disruptions in the adc gene itself, a second phenotypic marker of phase variation, decreased sensitivity to erythromycin, was analysed. This indicated that the difference in haemolytic activity of MLT5 is probably the result of a disruption of $a d c$, the adenylate cyclase toxin-haemolysin gene, which is in the same region as the Tn5 insert in this mutant, as no corresponding increase in erythromycin resistance occurred to indicate a shift of the bacteria from virulence to avirulence. The absence of any significant difference in sensitivity to human serum between the haemolytic and nonhaemolytic phenotypes of MLT5 also supports this hypothesis. Increases in production of nonhaemolytic variants by MLT2 and MLT3 which are accompanied by an increase in erythromycin resistance will require more detailed analysis of the $\operatorname{Tn} 5$-disrupted region for clarification of the situation but do implicate an association between $B$. pertussis LPS production and phase variation. Allen $\&$ Maskell (1996) described a partial homology between the $b p l l$ gene and $b v g S$. It would be intriguing if the Tn5 insert in MLT2 mapped to $b p l l$ and had an influence on phase variation from this location.

Studies of possible interactions between isolated LPS and associated molecules such as pertussis toxin have already been undertaken in several laboratories with 
encouraging results (Redhead \& Seagroatt, 1986; Sidey et al., 1989). Additional assays of biological alteration of whole-cell properties are presently underway and should expand our understanding of the relationship of LPS structure to outer membrane function and bacterial pathogenicity. We are particularly interested in whether the endotoxic properties of some of the LPS mutant strains have been altered enough to affect the local and systemic reactions associated with injection of the whole-cell vaccine.

The bacterial outer membrane is the dynamic template upon which the host-pathogen relationship evolves. LPS provides much of the 'framework' for this matrix. The creation and characterization of these mutants provide an opportunity by which the complete role of LPS in the pathogenicity and reactivity of the whole cell can be examined. Furthermore, these mutants provide a background in which LPS associations with other molecules can be studied. Secondary mutations could be used to define the nature of synergistic or associative relationships the LPS may have in vivo with other cell components such as pertussis toxin. Such interactions may be more important to infection and immunity than previously envisaged.

\section{REFERENCES}

Allen, A. \& Maskell, D. (1996). The identification, cloning and mutagenesis of a genetic locus required for lipopolysaccharide biosynthesis in Bordetella pertussis. Mol Microbiol 19, 37-52.

Archambault, D., Rondeau, P., Martin, D. \& Brodeur, B. R. (1991). Characterization and comparative bactericidal activity of monoclonal antibodies to Bordetella pertussis lipopolysaccharide A.J Gen Microbiol 137, 905-911.

Ausubel, F. M., Brent, R., Kingston, R. E., Moore, D. D., Seidman, J. G., Smith, J.A. \& Struhl, K. (1987). Current Protocols in Molecular Biology. New York: John Wiley.

Bannatyne, R. M. \& Cheung, R. (1984). Antibiotic resistance of degraded strains of Bordetella pertussis. Antimicrob Agents Chemother 25, 162-165.

Bass, J. W., Crast, F. W., Kotheimer, J. B. \& Mitchell, I. A. (1969). Susceptibility of Bordetella pertussis to nine antimicrobial agents. Am J Dis Child 117, 276-280.

Byrd, D. W., Roop, R. M., Veit, H. P. \& Schurig, G. G. (1991). Serum sensitivity and lipopolysaccharide characteristics in Bordetella bronchiseptica, B. pertussis and B. parapertussis. J Med Microbiol 54, 159-165.

Caroff, M., Chaby, R., Karibian, D., Perry, J., Deprun, C. \& Szabo, L. (1990). Variations in the carbohydrate regions of Bordetella pertussis lipopolysaccharides: electrophoretic, serological, and structural features. J Bacteriol 172, 1121-1128.

Chaby, R. \& Caroff, M. (1988). Lipopolysaccharides of Bordetella pertussis endotoxin. In Pathogenesis and Immunity in Pertussis, pp. 247-271. Edited by A. C. Wardlaw \& R. Parton. Chichester: John Wiley.

Cody, C. L., Baraff, L. J. \& Cherry, J. D. (1981). Nature and rates of adverse reactions associated with DPT and DT immunizations in infants and children. Pediatrics 68, 650-660.

Fernandez, R. C. \& Weiss, A. A. (1994). Cloning and sequencing of a Bordetella pertussis serum resistance locus. Infect Immun 62, $4727-4738$.
Gold, R. (1988). Pertussis vaccine: myths and realities. Can Fam Physician 34, 1167-1168.

Khelef, N., Bachelet, C., Vargaftig, B. B. \& Guiso, N. (1994). Characterization of murine lung infection after infection with parental Bordetella pertussis and mutants deficient in adhesins or toxins. Infect Immun 62, 2893-2900.

Laemmli, U. K. (1970). Cleavage of structural proteins during the assembly of the head of bacteriophage T4. Nature 227, 680-685.

Lasfargues, A., Caroff, M. \& Chaby, R. (1993). Structural features involved in the mitogenic activity of Bordetella pertussis lipopolysaccharides for spleen cells of $\mathrm{C} 3 \mathrm{H} / \mathrm{He} J$ mice. FEMS Immunol Med Microbiol 7, 119-130.

Lebbar, S., Caroff, M., Szabo, L., Merienne, C. \& Szilogyi, L. (1994). Structure of a hexasaccharide proximal to the hydrophobic region of lipopolysaccharides present in Bordetella pertussis endotoxin preparations. Carbohydr Res 259, 257-275.

Le Blay, K., Caroff, M., Blanchard, F., Perry, M. B. \& Chaby, R. (1996). Epitopes of Bordetella pertussis lipoplysaccharides as potenial markers for typing of islolates with monoclonal antibodies. Microbiology 142, 971-978.

LeDur, A., Chaby, R. \& Szabo, L. (1980). Isolation of two proteinfree and chemically different lipopolysaccharides from Bordetella pertussis phenol-extracted endotoxin. J Bacteriol 143, 78-88.

Liang-Takasaki, C.-J., Makela, P. H. \& Leive, L. (1982). Phagocytosis of bacteria by macrophages: changing the carbohydrate of lipopolysaccharide alters interaction with complement and macrophages. J Immunol 128, 1229-1235.

Makela, P. H. \& Stocker, B. A. D. (1984). Genetics of lipopolysaccharide. In Handbook of Endotoxin, vol. 1, Chemistry of Endotoxin, pp. 59-137. Edited by E. Th. Rietschel. Amsterdam: Elsevier.

Martin, D., Peppler, M. S. \& Brodeur, B. R. (1992). Immunological characterization of the lipopolysaccharide B band of Bordetella pertussis. Infect Immun 60, 2718-2725.

de Moissac, Y., Ronald, S. L. \& Peppler, M. S. (1993). The use of pulsed-field gel electrophoresis for the epidemiology of Bordetella pertussis in a whooping cough outbreak. J Clin Microbiol 32, $398-402$.

Nikaido, H. \& Vaara, M. (1985). Molecular basis of bacterial outer membrane permeability. Microbiol Rev 48, 1-32.

Osborne, M. J. (1968). Biochemical characterization of mutants of Salmonella typhimurium lacking glucosyl or galactosyl lipopolysaccharide transferases. Nature 217, 957-960.

Peppler, M. S. (1982). The isolation and characterization of isogenic pairs of domed hemolytic and flat nonhemolytic types of Bordetella pertusssis. Infect Immun 35, 840-851.

Peppler, M. S. (1984). Two physically and serologically distinct lipopolysaccharide profiles in strains of Bordetella pertussis and their phenotype variants. Infect Immun 43, 224-232.

Peppler, M. S. \& Schrumpf, M. E. (1984). Isolation and characterization of Bordetella pertussis phenotype variants capable of growing on nutrient agar: comparison with phases III and IV. Infect Immun 43, 217-223.

Redhead, K. \& Seagroatt, V. (1986). The effects of purified components of Bordetella pertussis in the weight gain test for the toxicity of pertussis vaccines. J Biol Stand 14, 57-65.

Roantree, R. J., Kuo, T. \& McPhee, D. G. (1977). The effect of defined lipopolysaccharide core defects upon antibiotic resistances of Salmonella typhimurium. J Gen Microbiol 103, 223-234.

Sambrook, J., Fritsch, E. F. \& Maniatis, T. (1989). Molecular 
Cloning: a Laboratory Manual, 2nd edn. Cold Spring Harbor, NY : Cold Spring Harbor Laboratory.

Sanderson, K. E., MacAlister, T., Costerton, J. W. \& Cheng, K.-J. (1974). Permeability of lipopolysaccharide-deficient (rough) mutants of Salmonella typhimurium to antibiotics, lysozyme, and other agents. Can J Microbiol 20, 1135-1145.

Sidey, F. M., Furman, B. L. \& Wardlaw, A. C. (1989). Effect of hyperreactivity to endotoxin on the toxicity of pertussis vaccine and pertussis toxin in mice. Vaccine 7, 237-241.

Southern, E. M. (1975). Detection of specific sequences among DNA fragments separated by gel electrophoresis. $J \mathrm{Mol}$ Biol $\mathbf{9 8 ,}$ 503-506.

Stainer, D. W. \& Scholte, M. J. (1971). A simple chemically defined medium for the production of phase I Bordetella pertussis. J Gen Microbiol 63, 211-220.
Stibitz, S. \& Garletts, T. L. (1992). Derivation of a physical map of the chomosome of Bordetella pertussis Tohama 1. J Bacteriol 174, 7770-7777.

Tsai, C. M. \& Frasch, C. E. (1982). A sensitive silver stain for detecting lipopolysaccharides in polyacrylamide gels. Anal Biochem 119, 115-119.

Weiss, A. A. \& Hewlett, E. L. (1986). Virulence factors of Bordetella pertussis. Annu Rev Microbiol 40, 661-686.

Weiss, A. A., Hewlett, E. L., Myers, G. A. \& Falkow, S. (1983). Tn5induced mutations affecting virulence factors of Bordetella pertussis. Infect Immun 42, 33-41.

Received 1 November 1996; revised 28 February 1997; accepted 4 March 1997. 